\title{
A Spoonful of Sugar:
}

\section{Understanding the Over-the-Counter Medication Needs and Practices of Older Adults}

\author{
Aqueasha M. Martin, Jessica N. Jones, Juan E. Gilbert \\ School of Computing \\ Clemson University \\ Clemson, SC USA \\ \{aqueasm,jnj, juan\}@clemson.edu
}

\begin{abstract}
Understanding the needs of various stakeholders throughout the design process is key for creating consumer health applications that are usable, effective, and useful for the people that use them. This paper discusses the findings of an exploratory study aimed at understanding the current practices, information needs, and challenges of older adults (65 years and older) when selecting over-the-counter (OTC) medication. The context of this study lies in understanding older adults with the purpose of identifying opportunities for a consumer health application to assist them with selecting an appropriate OTC medication based on their health history. This research contributes to the understanding of the current practices of older adults as related to OTC medication selection. In addition, this paper provides a discussion of opportunities for future applications to help facilitate this task for older adults.
\end{abstract}

Keywords- older adults; consumer-health applications; overthe-counter medication; medication

\section{INTRODUCTION}

Each year, thousands die or are injured due to adverse drug events (ADEs) resulting from taking medication [1, 2]. Medication interactions (ADEs resulting from a reaction to a medication) are considered to be the main cause of ADEs [3]. To this end, in recent years, several novel consumer health applications have emerged to support consumers in managing or understanding medication information. In addition, these applications have encouraged consumers to become more informed about the medication they take.

This research builds on previous work by providing insight on the processes, information needs, and challenges of older adults (65 years and older) when using over-the-counter (OTC) medication information to make a purchasing decision. OTC medication use, compared to prescription, is heavily dependent on a consumer's ability to understand and correctly use the OTC medication given their knowledge of their own health. Emphasis is placed on older adults because certain factors (e.g. lower health literacy levels, risk of chronic illness) increase with age, thereby increasing the risk of an $\operatorname{ADE}[4,5]$.

Although recent work has focused on understanding users in order to design various consumer health applications for medication, little has been done to understand the specific needs of older adults in order to effectively communicate information for the purpose of assisting them in the selection of an appropriate OTC medication. The authors define an appropriate medication as one that does not conflict with a chronic illness or other medication. Therefore, the goal of this exploratory study is to better understand the current challenges and needs of older adults in an effort to identify opportunities for a consumer health application to help facilitate this task.

\section{RELATED WORK}

Over the years, several researchers in the healthcare field have conducted studies to understand users with the goal of improving user interactions with medication information. In 1999, the Food and Drug Administration published the OTC Drug Facts regulation as a guide for makers of OTC medication to follow [6]. That same year, researchers published the results of a study examining users' OTC druglabeling format preferences [7]. Following this study, several studies were published that focused on different aspects of paper-based medication information design [7-10].

Similarly, researchers have used various techniques to better understand users with the goal of designing consumerbased applications to assist with different aspects of the medication regimen [11-19]. Seik and colleagues looked specifically at the needs of older adults to understand the requirements for a personal health application to help them manage medication [16]. Wilcox and colleagues examined the needs of cardiology patients to design an in-hospital application to provide them with information about their care (including medication and treatment) during their stay [17]. Neafsey and colleagues explored the needs of older adults to understand how to design an interactive educational intervention to warn about OTC medication and alcohol interactions [18].

Although previous work in this area has provided insight on the needs of different groups of users for different tasks related to medication, to the knowledge of the researchers, no study has focused on understanding the needs of older adults when they attempt to select an OTC medication. This work builds on previous literature by contributing to the understanding of older adults as related to selecting an OTC medication. Based on the findings, this research provides a discussion of opportunities for consumer health applications to assist in communicating OTC medication information to help facilitate the decisionmaking process.

\section{FIELD STUDY}

The study was conducted on-site at two locations within the local community.
A research team member met and

This research was funded in part by a Social Science Research Council Graduate Studies Enhancement Grant. 
conducted meetings with administrators at each location to explain the goals of the study, answer questions, obtain permission to recruit members, and obtain research site letters for the institutional review board (IRB) application. Proper IRB approval was obtained before the study began.

\section{A. Study Location and Participants}

The study was performed at two locations: a senior lifelong learning center and a local senior activity center. Participants of the study were members of one of the two organizations. Participants were recruited by email or through a verbal announcement at the center by one of the researchers. Participants in the study were required to be at least 65 years of age, purchase or select their own over-the-counter medication, and have purchased or selected for purchase at least one overthe-counter medication in the past year.

A total of 10 participants were recruited for the study $($ female $=7$, male $=3)$ with ages ranging from $66-82($ mean $=$ 74.2 , S.D. $=4.685$ ). Five participants were Caucasian and five participants were African-American. One participant had a middle school education, one earned a Master's degree, and one earned a Ph.D. Most participants had at least some college education $(\mathrm{n}=7)$.

All 10 participants indicated that they took medication (prescription and over-the counter). When asked how many medications they take on a daily or weekly basis, participant responses ranged from $3-12$ (mean $\sim 7$ ). In addition, all participants indicated that they had taken at least one over-thecounter medication within the last year $(\min =2$ daily, $\max =5$ daily). All participants reported that they primarily purchased their own OTC medication, however participants with a significant other reported that their spouse would sometimes purchase OTC medication for them.

\section{METHODS}

Semi-structured interviews were conducted with participants and all interviews were audio recorded and later transcribed for analysis. Data were analyzed for emerging themes to create an initial coding guide. Two researchers met several times to discuss emerging themes and make updates to the initial coding guide. Researchers later used the coding guide to independently and thematically code participant responses. The percent of agreement among researchers was greater that $80 \%$ for all categories, indicating a high-level of agreement among coders.

Interview questions were designed to better understand some of the current practices and challenges of older adults when choosing an OTC medication. These questions fit broadly into the two categories detailed below.

1) Current OTC Selection Practices: OTC medication, unlike prescription, does not require oversight from a healthcare provider and can be purchased at will by a consumer [20]. It was important to get a sense of older adults' attitudes towards OTC medication selection. Participants were therefore asked questions to understand their current selection process, the people involved, and the resources they consult.

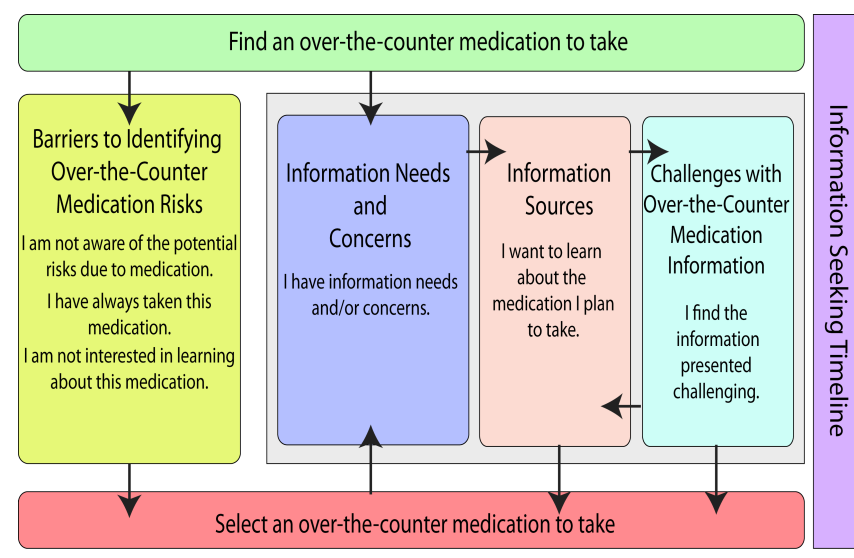

Figure 1. Older adults' over-the-counter medication selection process.

2) Challenges with OTC Medication Information: Questions in this category focused on understanding some of the challenges older adults face when using OTC medication information to make a decision to select or purchase. Participants were asked to describe any challenges they had with medication information in the past year. Participants were also given examples of OTC medication information (labels and page from WebMD) and asked to identify and discuss anything they found cumbersome or helpful about the information or the presentation of the information on the labels.

\section{FINDINGS}

Participants' responses to interview questions were categorized into high-level themes based on the thematic coding of the interview data. In interviews, participants described their current OTC medication selection processes at a conceptual level. In this process of selecting an OTC medication, older adults choose one or more paths that provide a basis for their information-seeking timeline (See Fig. 1). When searching for an OTC medication, the older adults either encounters a barrier that prevents him or her from identifying the benefits and risks of the OTC medication or is faced with information needs or concerns that trigger a cycle in which the older adult seeks information from information sources. In this cycle, if the older adult finds the information challenging, he or she may consult additional information sources until an OTC medication is selected. Additionally, once the older adult has selected an OTC medication to take, he or she may seek additional information regarding a medication that has been already selected. The steps in this process correspond to one of the key-themes identified and are detailed below.

\section{A. Information Seeking Timeline}

Of the participants that mentioned that they sought information about their OTC medications, some indicated that they would seek this information after first taking the OTC medication. This was due to various factors, including timing of scheduled visits with their doctors or having taken the medication before. For example, four participants mentioned that they felt comfortable asking their doctor about medications during regular visits. P8 stated, "If I have any questions [about an OTC medication], I ask the pharmacist and then at my next appointment with the doctor, I will ask the doctor." 


\section{B. Barriers to Identifying OTC Medication Risks}

The interviews revealed several potential barriers that may prevent older adults from identifying OTC medication risks. One participant expressed his perception that OTC medications generally could not do much harm. He stated, "You have a feeling, if you buy something over-the-counter, it's probably not going to kill you, right?"

Three participants mentioned that history or familiarity with a medication might influence whether or not someone seeks advice about an OTC medication before selecting. P1 stated, "The other thing is, if it's a person who's been around for decades, and decades, and decades ... and they've taken Advil their whole life, they're just going to grab a jar of Advil. You know, you sometimes develop an affinity for a certain pill that's over-the-counter."

Finally, six participants indicated they were generally not interested or ignored OTC medication information for various reasons. P6 stated, "I look at risks, but I sadly have grown to the point of almost ignoring it because they list so many risk and it seems though it's mostly just for liability reasons."

\section{Information Needs and Concerns}

Participants discussed several pieces of information they found important when making a decision about taking an OTC medication. The top information need discussed was medication use, mentioned by eight of the ten participants. Medication use was followed by interactions, mentioned by seven of the ten participants. At least two participants discussed brand of medication, side-effects/risks, dosage information/directions, ingredients, and price/cost each as important pieces of information for making a decision to purchase a medication. One participant discussed expiration date and another discussed the route (how the medication is administered) as an information need.

\section{Information Sources}

Participants indicated a variety of information sources for learning about OTC medications. Several participants expressed that they would consult a doctor or pharmacist about an OTC medication. For example, when asked about the OTC medication selection process, Participant 2 (P2) stated, "Well, if I am not sure, I just ask the druggist [pharmacist]." Additionally, P1 expressed concerns about asking the doctor or pharmacist about an OTC medication. She stated, "They [the doctors] are so busy now... The pharmacist is a little bit more available. I tend not to bother them if the store is busy or something." Several participants also mentioned consulting friends or family (word-of-mouth) or using the OTC medication label.

Other participants mentioned using some type of technology to help them learn about an OTC medication. For example, P5 stated, "Speaking for myself, I do have an app on my pad [iPad] that will give me the generic and the ... What is it when it's not generic, when there's a patented name?" However, when mentioned, the Internet was the primary source for searching for medication information. Most participants however, did not cite a technological source for learning about OTC medications. P1 provided an example of how she searched for medication information on the Internet, but later stated, "When I'm buying something over-the-counter, I usually don't go into this great detail."

\section{E. Challenges with OTC Medication Information}

One of the most common challenges discussed regarding OTC medication information was the layout and formatting of the text on the OTC packaging. Eight of the ten participants discussed challenges they had with either the size of the text or the organization of the information. P4 expressed frustration when searching for the dosage on the OTC medication packaging. He stated, "Where [is the] dosage? It's not here. Okay, you got to go on the other side and the dosage is buried in the middle of other things. 'Stop and ask your doctor if you vomit or something'. Well, I would like the directions [and] the dosage to be right up front at the beginning."

Another common complaint was with the language. Participants expressed that often times they did not understand the information on the label which made it difficult to use. P1 stated, "The other thing I see is a problem ... I think they said, 'Don't take this if you are taking other drugs containing prescription or non-prescription NSAIDs'... I wouldn't know what an NSAID is unless I Googled it."

Participants also discussed challenges with the amount and kind of information presented. P6 stated, "I think too that it's so complex. There's so much to try and read and absorb off that label..." He later suggested that some types of information were not there or not apparent.

\section{DISCUSSION AND CONCLUSIONS}

The findings of this study provide a better understanding of the current practices of older adults related to OTC medication selection and reveal several opportunities for technology to assist in this task. Although some participants in this study did not cite a technological resource for learning about their OTC medication, their non-use or non-adoption of current technology may be tied to the perceived benefit (i.e. usefulness) or effectiveness of current technology for completing the targeted task [21].

For example, consistent with other studies, participants in this study expressed challenges with the formatting and layout of OTC medication information [7-10]. Although prior research has produced guidelines for label formatting and layout, understanding that font size is still a concern among older adults presents the opportunity for designers to provide technology that can address this concern (e.g. tools that provide the same information with larger font) and highlight a benefit that is currently not addressed through labeling.

Given that older adults are at a greater risk for adverse drug events due to medication, it is imperative that they are aware of the potential benefits and risks $[4,5]$. Upon this realization, they should be able to clearly see, find, and understand the information provided to make an informed decision. A consumer health application, therefore, has the opportunity to address some of the limitations of labeling, which may help better support older adults in the process of selecting appropriate OTC medications. Furthermore, careful 
technology design may also increase awareness of OTC medication risks, which can incentivize the use of such technology among the older adult population [21]. A discussion of opportunities is provided below.

\section{A. Technology to Support Language Simplification}

Regardless of education level or expertise, understanding health information is a challenge that is faced by many in the population [4]. Participants of this study also expressed challenges with understanding some of the medical terminology used on the label. Technology may therefore be useful to bridge the gap between the required medical terminology used on the label for accuracy and the layperson terminology desired by the consumers for making a decision. Using plain language guides in the design or automated techniques (e.g. text simplification) for simplifying language in the application may greatly improve the usability of the information for any user, not just older adults.

\section{B. Technology to Support Awareness}

The findings of this study also imply that some older adults may not be aware of the potential risks of OTC medication. Depending on the type of application being developed, designers may also consider integrating elements of persuasion into their applications. Persuasive technology design aims to change the attitudes and/or behaviors of users through one of seven persuasion techniques: reduction, tunneling, tailoring, suggestion, self-monitoring, surveillance, or conditioning [22]. Older adults in this study mentioned challenges that can potentially be addressed by one or more of these techniques. For example, reduction persuades by simplifying the steps a user takes to complete a task. If the task of finding relevant information can be simplified through technology design, older adults may change their attitudes toward seeking OTC information in the future.

\section{LIMITATIONS AND FUTURE WORK}

Though interviews were conducted until no new data emerged, the number of people interviewed limits the study. Further work is needed to determine if the findings can be generalized to the larger population of older adults. Future studies will be conducted to confirm the usefulness of the opportunities described and to further examine ways of addressing the concerns through technology.

\section{REFERENCES}

[1] DA DeWalt, "Ensuring safe and effective use of medication and health care: perfecting the dismount.," JAMA : the journal of the American Medical Association, vol. 304, no. 23, pp. 2641-2, Dec. 2010.

[2] Committee on Quality of Healthcare America and Institute of Medicine, To Err Is Human: Building a Safer Health System. National Academies Press, 2000, p. 287.

[3] L. C. Curry, C. Walker, M. O. Hogstel, and P. Burns, "Teaching older adults to self-manage medications: preventing adverse drug reactions.," Journal of gerontological nursing, vol. 31, no. 4, pp. 32-42, Apr. 2005.

[4] National Center for Education Statistics. (2006, September). The Health Literacy of America's Adults: Results From the 2003 National Assessment of Adult Literacy [Online]. Available: http://nces.ed.gov/pubs2006/2006483.pdf
[5] Centers for Disease Control. (2011, May 11). Healthy Aging [Online]. Available: http://www.cdc.gov/chronicdisease/resources/publications/AAG/aging.h $\underline{\mathrm{tm}}$

[6] United States Food and Drug Administration. (2009, May 28). Information for Consumers- OTC Drug Facts Label [Online]. Available: http://www.fda.gov/Drugs/ResourcesForYou/Consumers/ucm143551.ht $\underline{\mathrm{m}}$

[7] W. J. Vigilante and M. S. Wogalter, "Over-The-Counter (OTC) Drug Labeling: Format Preferences," Proceedings of the Human Factors and Ergonomics Society Annual Meeting, vol. 43 , no. 2 , pp. 103-107, Sep. 1999.

[8] H. A. Klein and J. J. Isaacson, "Making Medication Instructions Usable," Ergonomics in Design: The Quarterly of Human Factors Applications, vol. 11 , no. 2 , pp. 7-12, Apr. 2003.

[9] D. G. Morrow, M. Weiner, J. Young, D. Steinley, M. Deer, and M. D. Murray, "Improving medication knowledge among older adults with heart failure: a patient-centered approach to instruction design.," The Gerontologist, vol. 45, no. 4, pp. 545-52, Aug. 2005.

[10] S. S. Sansgiry and M. D. Pawaskar, "Obstruction of critical information on over-the-counter medication packages by external tags.," The Annals of pharmacotherapy, vol. 39, no. 2, pp. 249-54, Feb. 2005.

[11] T. W. Bickmore, L. M. Pfeifer, and B. W. Jack, "Taking the time to care," in Proceedings of the 27th international conference on Human factors in computing systems - CHI 09, 2009, p. 1265.

[12] M. Hoogendoorn, M. C. A. Klein, and B. Mosch, "Online application for simulating intelligent support for medicine intake," in Proceedings of the 1st ACM international conference on PErvasive Technologies Related to Assistive Environments - PETRA '08, 2008, p. 1.

[13] I. Qudah, P. Leijdekkers, and V. Gay, "Using mobile phones to improve medication compliance and awareness for cardiac patients," in Proceedings of the 3rd International Conference on PErvasive Technologies Related to Assistive Environments-PETRA '10, 2010, p. 1.

[14] R. de Oliveira, M. Cherubini, and N. Oliver, "MoviPill," in Proceedings of the 12th ACM international conference on Ubiquitous computing Ubicomp '10, 2010, p. 251.

[15] J. M. Silva, A. Mouttham, and A. El Saddik, "UbiMeds," in Proceedings of the 1st ACM SIGMM international workshop on Media studies and implementations that help improving access to disabled users MSIADU '09, 2009, p. 71.

[16] K. A. Siek, D. U. Khan, S. E. Ross, L. M. Haverhals, J. Meyers, and S. R. Cali, "Designing a personal health application for older adults to manage medications: a comprehensive case study.,". Journal of medical systems, vol. 35, no. 5, pp. 1099-121, Oct. 2011.

[17] L. Wilcox, S. Feiner, A. Liu, S. Restaino, S. Collins, and D. Vawdrey, "Designing inpatient technology to meet the medication information needs of cardiology patients," in Proceedings of the 2nd ACM SIGHIT symposium on International health informatics - IHI '12, 2012, p. 831.

[18] P. J. Neafsey, Z. Strickler, J. Shellman, and V. Chartier, "An interactive technology approach to educate older adults about drug interactions arising from over-the-counter self-medication practices.," Public health nursing (Boston, Mass.), vol. 19, no. 4, pp. 255-62.

[19] M. Harjumaa, M. Isomursu, S. Muuraiskangas, and A. Konttila, "HearMe: A touch-to-speech UI for medicine identification," 20115 th International Conference on Pervasive Computing Technologies for Healthcare PervasiveHealth and Workshops, pp. 85-92, 2011.

[20] T. R. Covington, "Nonprescription drug therapy: issues and opportunities.," American journal of pharmaceutical education, vol. 70, no. 6, p. 137, Dec. 2006.

[21] A. Melenhorst, W. Rogers, and D. Bouwhuis, "Older Adults' Motivated Choice for Technological Innovation: Evidence for Benefit-Driven Selectivity," Journal of Psychology and Aging, vol. 21, no. 1, pp. 190195, Mar. 2006.

[22] B.J. Fogg, Persuasive Technology: Using Computers to Change What We Think and Do. Morgan Kaufmann, 2003. 